http://dx.doi.org/10.35381/e.k.v4i1.1456

\title{
Baamboozle como estrategia de enseñanza de la tabla periódica en estudiantes de tercero de Bachillerato
}

\section{Baamboozle as a teaching strategy of the periodic table in third year high school students}

\author{
Mónica Patricia Arce-Becerra \\ monica.arce.87@est.ucacue.edu.ec \\ Universidad Católica de Cuenca, Azogues \\ Ecuador \\ https://orcid.org/0000-0003-2266-3940 \\ Darwin Gabriel García-Herrera \\ dggarciah@ucacue.edu.ec \\ Universidad Católica de Cuenca, Azogues \\ Ecuador \\ https://orcid.org/0000-0001-6813-8100 \\ Juan Carlos Erazo-Álvarez \\ jcerazo@ucacue.edu.ec \\ Universidad Católica de Cuenca, Cuenca \\ Ecuador \\ https://orcid.org/0000-0001-6480-2270
}

Recepción: 10 de agosto 2021

Revisado: 15 de septiembre 2021

Aprobación: 15 de noviembre 2021

Publicación: 01 de diciembre 2021 


\title{
RESUMEN
}

Se planteó la investigación para analizar como la implementación de la herramienta Baamboozle contribuye a la enseñanza de la tabla periódica en estudiantes de tercero de secundaria; para este estudio se planteó una metodología cuasiexperimental, de cohorte longitudinal; después de aplicar la herramienta los resultados de indicaron que el uso de la herramienta digital favorece la enseñanza y comprensión de la tabla periódica. Los estudiantes presentaron predisposición para el aprendizaje del tema, por lo tanto, el uso de herramientas digitales mejora el aprendizaje de la tabla periódica y además permiten atraer a los estudiantes al aprendizaje de esta herramienta química.

Descriptores: Química; propiedad química; educación tradicional. (Palabras tomadas del Tesauro UNESCO).

\begin{abstract}
Se planteó la investigation para analizar como la implementación de la herramienta Baamboozle contribuye a la enseñanza de la tabla periodic en estudiantes de tercero de secondaryia; a cuasiexperimental methodology, of a longitudinal cohort; después de aplicar la herramienta los results de indicaron que el uso de la herramienta digital favorece la enseñanza y comprension de la tabla periódica. Los estudiantes presentaron predisposición para el aprendizaje del tema, por lo tanto, el uso de herramientas digitales mejora el aprendizaje de la tabla periódica y además permiten atraer a los estudiantes al aprendizaje de esta herramienta chemistry.
\end{abstract}

Description: Chemistry; chemical properties; traditional education. (UNESCO Palabras tomadas del Tesauro). 


\section{INTRODUCCIÓN}

El proceso educativo no debe ser calificado como una transferencia y recolección de conocimientos, sino más bien debe ayudar a la formación de ciudadanos competentes para incorporarse a la sociedad; en este sentido es importante un cambio en el rumbo educativo, estableciendo métodos creativos de enseñanza enfocados en solucionar las diferentes problemáticas que ocurren en la vida diaria. De tal forma, la instrucción de las ciencias químicas es una de las dificultades que enfrenta actualmente la educación virtual; según (Tejada-Tovar et al. 2013), la enseñanza y aprendizaje de química consecutivamente ha demostrado dificultades tanto en el ámbito pedagógico como didáctico, la mayoría de estudiantes no manifiestan interés por el aprendizaje de esta asignatura debido grado de abstracción que presentan ciertas temáticas.

En relación a la situación que se plantea, (Domènech-Casal, 2019), establece que en España los alumnos de secundaria presentan inconvenientes principalmente en la comprensión y análisis de las carcateristicas de los elementos de la tabla periódica, además se indica que la metodología empleada por los docentes no estimula a los estudiantes para el aprendizaje.

Adicionalmente (Ramos-Mejía, 2020), plantea que en México el abordaje de la temática de la tabla periódica se lo hace en base a la clase tradicionalista mediante la continua repitición oral o escrita de los distintos elementos químicos, por lo tanto no existe interés o motivación por parte del alumnado hacia el aprendizaje, ocasionando reiteradamente el olvido de lo aprendido, generando estudiantes pasivos con poca afición para el aprendizaje. Por otro lado los juegos didácticos contribuyen al aprendizaje de la periodicidad de los elementos químicos, pues como lo establece (Marcano-Godoy, 2020), la incorporación de actividades lúdicas como estrategia de enseñanza cambia el esquema tradicionalista de educación, logrando en los estudiantes una mayor compresión de la simbología quimica y características de los elementos químicos. 
En base a lo expuesto y a la problemática que representa el aprendizaje de la tabla periódica se plantea la presente invetsigación para analizar cómo el uso de la herramienta Bamboozle como estrategia didáctica puede aportar a la enseñanza de la tabla periódica en los estudiantes de secundaria de la Unidad Educativa Guachapala del cantón Guachapala de la provincia del Azuay-Ecuador.

\section{Referencial teórico}

En el proceso de enseñanza de las ciencias exactas como química es importante el empleo de estrategias y metodologías que faciliten a los alumnos entender los conceptos de forma activa mediante la interacción con el estudiante, de tal manera que el uso de herramientas digitales se han convertido en las nuevas estrategias de enseñanza de las diferentes temáticas que engloba esta asignatura, pues mediante su empleo se puede realizar actividades lúdicas que permiten la mejor compresión de la tabla periódica.

Así, en la investigación desarrollada por (López-Guerrero et al. 2018), en España concluye que los estudiantes de secundaria tienen un mayor grado de comprensión de la tabla periódica mediante el uso de simuladores virtuales, además los resultados de este estudio muestra que el uso de estas herrramientas digitales mejora y perfecciona la explicación tradicional realizada por el educador en el aula de clase; mejorando el rendimiento de los estudiantes.

Del mismo modo el trabajo realizado por (Plutin-Pacheco \& García-López, 2016), en Cuba establece que la implementación de actividades lúdicas mediante el uso de herramientas digitales permiten realizar una esquematización de los contenidos teóricos de la tabla periódica mejorándose así el rendimiento académico de la asignatura de química en comparación con el rendimiento académico en años anteriores, además el estudio establece que el empleo de juegos permite la inclusión y la participación de los estudiantes en el proceso de aprendizaje, manteniéndose una enseñanza activa de la simbología y conceptos relacionados con tabla periódica. 
En este sentido el trabajo realizado por (Castillo et al. 2017), en Venezuela confirma que el uso de las herramientas digitales para la preparación de juegos estimula el aprendizaje de la tabla periódica y a la vez facilita al docente el proceso de evaluación de los alumnos y también se puede tener acceso continuo a las actividades diseñadas, generándose así un proceso de retroalimentación del aprendizaje de la tabla periódica y los elementos químicos.

Por otro lado, la educación virtual ha encaminado a los docentes de química a implementar nuevas estrategias de enseñanza mediante el empleo de las herramientas digitales, en tal sentido (Baptista- Lucio et al. (2020) concluye en su estudio realizado en México que los docentes durante las clases virtuales han sido creativos y han ido cambiando las estrategias didácticas de enseñanza de química, por lo tanto los profesores han empleado desde los textos escolares hasta una gran variedad de recursos y plataformas digitales para lograr el aprendizaje significativo de las ciencias químicas.

\section{Enseñanza de la tabla periódica de los elementos químicos}

La enseñanza de la simbología y propiedades de la tabla periódica es compleja pues según lo planteado por (Écija-Conesa, 2019), la mayoría de estudiantes tiene poca predisposición y falta de interés por el aprendizaje de la tabla periódica. En este sentido los docentes se han visto en la necesidad de cambiar esta situación mediante el uso de técnicas nuevas que reemplace la enseñanza tradicional.

Es importante señalar que la tabla periódica constituye una herramienta básica para la enseñanza de química pues constituye una representación que organiza a los elementos químicos en orden asecendenete según el número atómico. Además en esta herramienta se representa varias propiedades de los elementos químicos como: masa atómica, número atómico, tipo de elemento, distribución de partículas electrónicas. También en este instrumento se encuentran clasificados los elementos químicos según sus caracteristicas en metales, no metales, metaloides y gases nobles. 
Según la investigación realizada por (Bernal-Márquez et al. 2012), se establece que en general el tema no suele generar una actitud positiva hacia el aprendizaje; además la dificultad de aprendizaje se debe a la complejidad de las nociones químicas que están implícitas en la tabla periódica como número atómico, masa atómica, periodicidad de los elementos químico; es importante señalar que la mayoría de las dificultades de enseñanza se debe a la incorrecta pedagogía y metodología empleada por los docentes durante este tema.

Además, (Franco-Mariscal y Oliva-Martínez, 2012), señalan que el proceso de planificación que realiza el docente se basa en un currículo sobrecargado en el que se plantean tres momentos para el desarrollo de la clase: anticipación, construcción y consolidación; esta situación no le permite al maestro realizar clases innovadoras que faciliten los procesos de aprendizaje en los alumnos.

Es importante que se realice un cambio significativo en los procesos de enseñanza de química de tal manera que se motive en los estudiantes a desarrollar actitudes que predispongan al aprendizaje de las ciencias químicas, especialmente la tabla periódica; en este sentido los juegos tienen un papel importante en el desarrollo del aprendizaje de la tabla periódica, según (Bernal-Márquez et al. 2012), la formación de palabras, crucigramas, adivinanzas favorecen a memorizar los símbolos de los elementos químicos y comprender las nociones químicas que se relacionan con la tabla periódica, además el empleo de herramientas digitales permite afianzar el aprendizaje de la disposición de los elementos y la simbología química, permitiendo que el estudiantado se familiarice con la estructura, características y símbolos de la tabla periódica

\section{Herramientas digitales para la enseñanza de la tabla periódica}

En el estudio realizado por (Rojas-Viteri et al. 2021), en Ecuador se establece que la mayor parte de los estudiantes concuerdan en que las herramientas digitales como Kahoot o Baamboozle les permiten realizar un repaso de los contenidos revisados de las 
diferentes áreas incluyendo el área química, además la investigación indica que los alumnos tienen mayor motivación para el aprendizaje al emplear estas herramientas generando una mayor intervención de los educandos en la sala de clase. En este sentido es importante la implementación de herramientas para la motivar a los estudiantes, pues en lo concerniente a educación siempre es importante la innovación y con los beneficios de las herramientas digitales se puede generar nuevas rutas y esquemas de enseñanza para el alumando.

\section{METODOLOGÍA}

En este estudio se planteó una investigación experimental, pues según lo establecido por (Hernández-Sampiere et al. 2014), la investigación cuasi experimental consiste en realizar intervenciones para analizar las variables del estudio, en este sentido se aplicó como estrategia de enseñanza de la tabla periódica la herramienta Baamboozle; en relación al paradigma epistemológico metodológico fue cuantitativo pues se valoró mediante escala Likert las variables establecidas para el estudio.

El estudio fue de cohorte longitudinal pues los datos se recolectarón en dos momentos; en el tercero de secundaria paralelo $A$ se realizó una clase tradicional sin el empleo de la herramienta Bamboozle; en el tercero de secundaria B se realizó una clase empleando estrategias innovadoras y se implementó la herramienta Baamboozle para la enseñanza de la tabla periódica. La población estuvo conformada por los estudiantes de la Unidad Educativa Guachapala, se realizó un muestreo aleatorio estratificado y se obtuvo una muestra de 42 estudiantes distribuidos en dos paralelos de tercero de bachillerato.

La investigación fue de campo, se realizó en el cantón Guachapala de la provincia del Azuay, se aplicó una ficha de observación estructurada con cinco preguntas, este instrumento se elaboró de forma manual y fue validado por el coeficiente Alfa de Cronbach con un valor de 0,969 y al mismo tiempo se realizó una validación del instrumento por expertos aplicando el Método Delfi. 
Las variables analizadas en la investigación fueron: participación en clase, predisposición para el aprendizaje, nivel de atención a la clase, comprensión de conceptos teóricos, aprendizaje de simbología de elementos químicos, la ficha de observación contenía los siguientes niveles de medición: muy frecuentemente, frecuentemente, a veces, nunca y casi nunca.

Para el análisis estadístico de las variables se empleó el programa Statiscal Package for Social Sciences [SPSS] y se aplicó una estadísitica inferencial para el análisis de los resultados en los diferentes grupos en los que se realizó la investigación. Es importante establecer que se elaboró un consentimiento informado para establecer la participación de los estudiantes en la investigación así como la utilización de los datos recolectados durante la investigación para la elaboración y publicación de los resultados fruto de la investigación realizada.

\section{RESULTADOS}

En relación al análisis de resultados se aplicó una ficha de observación a 22 estudiantes de tercero de secundaria paralelo A y 19 estudiantes de tercero de secundaria paralelo $B$, para comprobar la fiabilidad del instrumento empleado se empleó el programa SPSS Para comprobar la hipótesis se aplicó la prueba T-Student para relacionar las variables categóricas y comprobar que el uso de la herramienta Baamboozzle como estrategia de enseñanza contribuye al aprendizaje de la tabla periódica, se estableció p menor a 0.05 se considera hipótesis nula y p mayor a 0.05 para que la hipóteisis tenga validez.

Se aplicó una prueba de normalidad para establecer si las variables que intervienen en el estudio son paramétricas o no paramétricas. En este sentido las variables: participación del estudiante dentro de la clase, predisposición para el aprendizaje de la tabla periódica, nivel de atención en la hora de clase, comprensión de los conceptos de la tabla periódica, facilidad de aprendizaje de símbolos de elementos químicos son paramétricas pues el 
valor de la prueba de normalidad Shapiro-Wilk para 41 datos recolectados fue menor a 0.05, $(p<0.05)$.

\section{Tabla 1.}

Resultados totales de grupo control y grupo experimental.

\begin{tabular}{|c|c|c|c|c|c|c|c|c|c|c|}
\hline & \multicolumn{2}{|c|}{ Part. } & \multicolumn{2}{|c|}{ Pred. } & \multicolumn{2}{|c|}{ Nivel de atención } & \multicolumn{2}{|c|}{ Compr. conceptos } & \multicolumn{2}{|c|}{$\begin{array}{c}\text { Apren. Simb. Tab } \\
\text { Periodica. } \\
\text { Grupo }\end{array}$} \\
\hline & Control & Exper. & Control & Exper. & Control & Exper. & Control & Exper. & Control & Exper. \\
\hline $\mathrm{N}$ & 22 & 19 & 22 & 19 & 22 & 19 & 22 & 19 & 22 & 19 \\
\hline Media & 2.50 & 4.37 & 2.36 & 4.63 & 2.32 & 4.32 & 1.82 & 4.21 & 1.82 & 4.11 \\
\hline $\begin{array}{c}\text { Desviación } \\
\text { estándar }\end{array}$ & 0.598 & 0.496 & 0.727 & 0.496 & 0.646 & 0.478 & 0.733 & .419 & 0.795 & 0.315 \\
\hline $\begin{array}{l}\text { Media de } \\
\text { error } \\
\text { estándar }\end{array}$ & 0.127 & 0.114 & 0.155 & 0.114 & 0.138 & 0.110 & 0.156 & 0.096 & 0.169 & 0.72 \\
\hline Sig. Bilateral & 0.000 & 0.000 & 0.000 & 0.000 & 0.000 & 0.000 & 0.000 & 0.000 & 0.000 & 0.000 \\
\hline
\end{tabular}

Fuente: Encuesta.

\section{Leyenda}

Part: participación en clase

Pred: predisposición para el aprendizaje

Compr.: Comprensión de conceptos teóricos

Apre. Simb. Tab. Periódica.: Aprendizaje de símbolos de la tabla periódica

En la tabla 1 se aprecia los resultados de la investigación y se puede establecer que en relación a la participación en clase en el grupo control en el cual no se empleó la herramienta Baamboozle la media es de 2.50 y en el grupo experimental al cual se usó la herramienta Baamboozle la media es de 4.37, con este resultado se pudo demostrar que los estudiantes presentaron mayor participación dentro de la hora de clase al 
momento de emplear estrategias innovadoras para la enseñanza de la tabla periódica, esta diferencia se afirma con el índice obtenido en la prueba $T(0.000)$, por lo tanto la hipótesis es afirmativa.

En lo relacionado a la predisposición al aprendizaje en donde el estudiante se siente atraído por la enseñanza de la tabla periódica la media en el grupo control es de 2.36 y la media del grupo experimental es de 4.63, con este resultado se puede notar que el empleo de la herramienta Baamboozle influye en el ánimo del alumno para conseguir una actitud positiva para el aprendizaje de la tabla periódica, alcanzado un índice de (0.000) en la prueba $T$ esto confirma que un cambio de estrategia de enseñanza predispone al aprendizaje de la tabla periódica. En cuanto al nivel de atención durante la hora de clase la media en el grupo control es de 2.32 y en el grupo experimental en el cual se empleó la herramienta Baamboozle es de 4.32, obteniéndose en la prueba T un índice de (0.000), con este resultado se confirma que los usos de estrategias innovadoras mejoran y mantienen la atención del estudiante dentro de la hora de clase.

En la comprensión de conceptos la media en el grupo control es de 1.82 y en el grupo experimental es de 4.21, en la prueba T el índice es (0.000) por lo tanto el empleo de la herramienta Baamboozle señala un cambio en la enseñanza que permite a los alumnos comprender los conceptos teóricos de la tabla periódica. Finalmente, en el aprendizaje de simbología de elementos químicos la media en el grupo control es de 1.82 y en el grupo experimental es de 4.11, este cambio se generó pues se combinó la herramienta Baamboozzle y la formación de palabras con los símbolos de los elementos químicos lo cual generó que el aprendizaje no se genere mediante la repetición oral y memorística de los símbolos y elementos químicos, en tal sentido en la prueba T se obtuvo un índice de (0.000) lo cual confirma que hubo un cambio significativo en el aprendizaje.

En este sentido el trabajo realizado por (Castillo et al. 2017), en Venezuela confirma que el uso de las herramientas digitales para la preparación de juegos estimula el aprendizaje de la tabla periódica y a la vez facilita al docente el proceso de evaluación de los alumnos 
y también se puede tener acceso continuo a las actividades diseñadas, generándose así un proceso de retroalimentación del aprendizaje de la tabla periódica y los elementos químicos.

Además, como lo indica (Basulto-Lemus et al. 2006), el impacto que ha causado la tecnología en el área educativa ha originado la creación de nuevas metodologías para el desarrollo de las clases y las herramientas digitales han contribuido en mejorar la enseñanza de química facilitando el aprendizaje de conceptos y símbolos de elementos químicos que muchas veces son difíciles de comprender y se ha empleado la enseñanza tradicional mediante el empleo de la memoria; esta situación no genera un aprendizaje significativo en el estudiante.

\section{PROPUESTA}

Para la implementación de la herramienta Baamboozle como estrategia para la enseñanza de la tabla periódica en tercero de secundaria se estableció la propuesta IDE, la cual se fundamenta en los siguientes aspectos: información general, desarrollo, evaluación; logrando de esta manera un aprendizaje significativo mediante la innovación del proceso de enseñanza. 


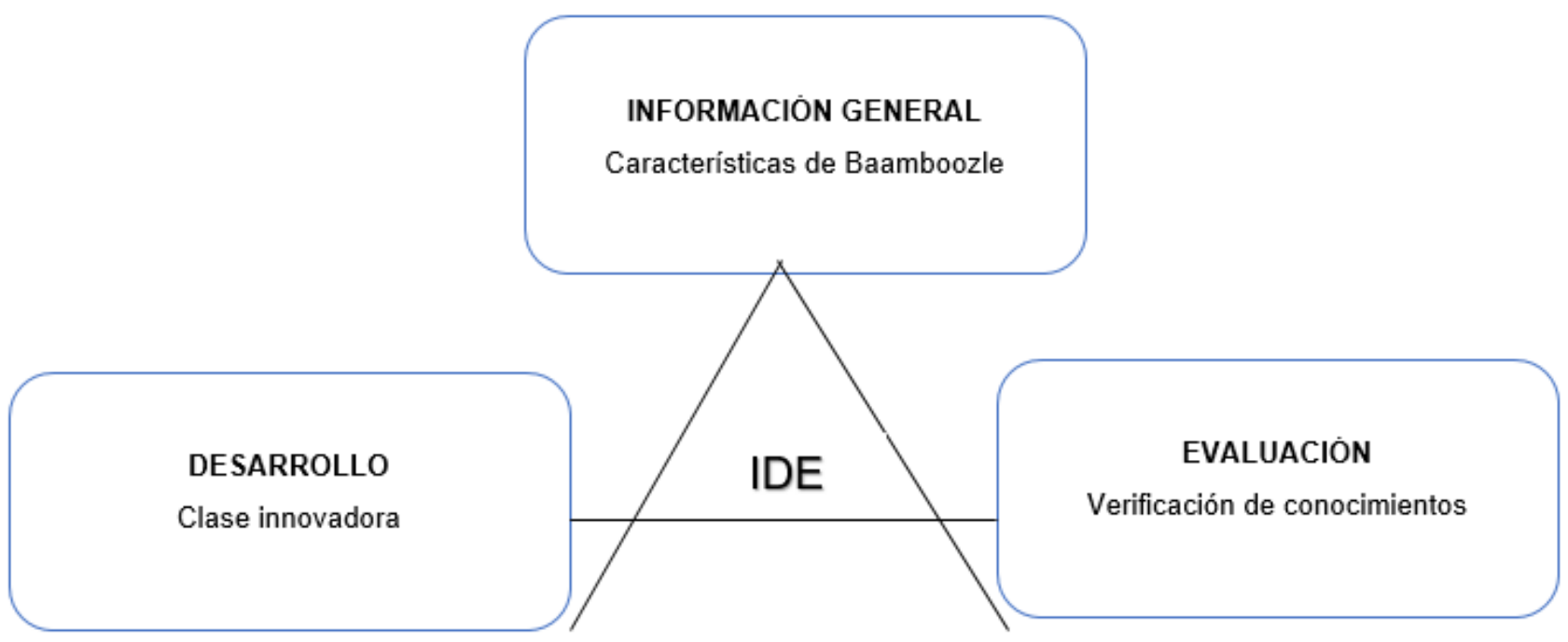

Figura 1. Esquema para implementación de herramienta Baamboozle en tercero de secundaria.

Elaboración: Los autores.

Información general: se realizó una capacitación a los estudiantes de tercero de secundaria sobre el manejo de la herramienta Baamboozle, se indicó las características y los beneficios de la herramienta Baamboozle, se procedió a realizar una demostración de las actividades que se pueden realizar y el modo de uso de la herramienta.

Desarrollo: durante esta etapa se elaboró la planificación microcurricular para proceder a impartir la clase con el empleo de la herramienta; la clase se desarrolló estableciendo los tres momentos: anticipación, construcción y consolidación; durante la anticipación se presentó el título de la clase La Tabla Periódica como un rompecabezas y se solicitó a los estudiantes que armen el título.

Seguidamente en el momento de la construcción se aplicó la herramienta Baamboozle, para lo cual se requirió formar dos equipos con los estudiantes, la herramienta plantea una serie de tarjetas las cuales tuvieron preguntas para el desarrollo de la clase, las 
preguntas para la comprensión de conceptos teóricos estaban diseñadas mediante adivinanzas y para el aprendizaje de los símbolos químicos las preguntas tenían palabras formadas con los símbolos de los elementos químicos. Los estudiantes de cada equipo tuvieron la oportunidad de escoger el número de pregunta y responderla, al final se estableció un puntaje que indicó al equipo ganador.

En la consolidación se dialogó con los alumnos sobre el uso de la herramienta y las actividades propuestas y se retroalimentó los conocimientos mediante el empleo de la herramienta Baamboozle.

Evaluación: se realizará mediante una ficha de observación para analizar la facilidad de aprendizaje de conocimientos teóricos de la tabla periódica, también se observó la predisposición, participación en clase, nivel de atención y aprendizaje de símbolos de la tabla periódica.

\section{CONCLUSIONES}

El uso de herramientas digitales predispone a los estudiantes para el aprendizaje de química, principalmente de la tabla periódica, por lo tanto, es importante el empleo de estrategias innovadora para captar la atención de los estudiantes y mejorar el proceso de enseñanza de las ciencias químicas.

El empleo de Baamboozle como estrategia de enseñanza mejora la predisposición de los estudiantes para el aprendizaje, el impacto del uso de esta herramienta fue positivo pues también mejoró la atención de los estudiantes durante la hora de clase, en este sentido los estudiantes no estaban distraídos mejorando de esta manera la comprensión de los conceptos teóricos de la tabla periódica.

La herramienta Baamboozle permite el desarrollo de actividades lúdicas como adivinanzas o formación de palabras, en este sentido el empleo de estas actividades para 
el estudio de conceptos teóricos permitió una mejor comprensión logrando un aprendizaje significativo en los estudiantes.

Es importante implementar durante las clases distintas herramientas digitales para facilitar el aprendizaje de los conceptos químicos, motivando de esta manera un aprendizaje divertido evitando la clase tradicionalista.

\section{FINANCIAMIENTO}

No monetario.

\section{AGRADECIMIENTOS}

A la Universidad Católica de Cuenca; por impulsar el desarrollo de este proceso investigativo desde la praxis educativa.

\section{REFERENCIAS CONSULTADAS}

Baptista-Lucio, P., Almazán-Zimerman, A., Loeza-Altamirano, C. A., López-Alcaraz, A. V., \& Cárdenas-Dominguez, J. L. (2020). Encuesta Nacional a Docentes ante el COVID-19. Retos para la educación a distancia [National Survey to Teachers Facing COVID-19. Challenges for Distance Education]. Revista Latinoamericana de Estudios Educativos, 50, 41-88. https://doi.org/10.48102/rlee.2020.50.especial.96

Basulto-Lemus, Y., Moreno-Toiran, G., Lahens-Calderón, D., \& Bernal-Medina, M. (2006). Las clases de química asistidas por software educativos: una perspectiva actual en las universidades pedagógicas [Chemistry classes assisted by educational software: a current perspective in pedagogical universities]. Revista Cubana de Química, XVIII(2), 160-166. https://www.redalyc.org/pdf/4435/443543704052.pdf

Bernal-Márquez, S., Oliva-Martínez, J. M. \& Franco-Mariscal, A. J. (2012). Una revisión bibliográfica sobre el papel de los juegos didácticos en el estudio de los elementos químicos. Primera parte: los juegos al servicio del conocimiento de la tabla periódica [A bibliographic review on the role of educational games in the study]. Educación Química, 23(3), 338-345. https://doi.org/10.1016/s0187-893x(17)30118-0 
Castillo, W., Martínez, F., Álamo, L. M., Sojo, V., Ramírez, B., Peraza, A., Rojas, L., Sánchez, M., Echeverría, M., Alfonzo, F., Rondón, P., Martínez, M., \& Ruette, F. (2017). EduQuim, una herramienta computacional para el aprendizaje y la enseñanza de química en la escuela secundaria [EduQuim, a computational tool for learning and teaching Chemistry in high school]. Educación y Educadores, 9(1), 127141. https://n9.cl/9refb

Domènech-Casal, J. (2019). Retorno a karlsruhe: una experiencia de investigación con la tabla periódica para aprender la estructura y propiedades de los elementos químicos. [Return to Karlsruhe: a research experience with the periodic table to learn the structure and properties of . Revista Eureka Sobre Enseñanza y Divulgación de Las Ciencias., 16(1), 1201-1219. https://doi.org/10.25267/rev eureka ensen divulg cienc.2019.v16.i1.1201

Écija-Conesa, A. (2019). El ministerio de la química: propuesta gamificadora para la enseñanza de la física y la química en el tercer curso de la educación scundaria obligatoria [The ministry of chemistry: gamification proposal for the teaching of physics and chemistry in the th [Universidad Católica de Murcia]. https://n9.cl/x9p0q

Franco-Mariscal, A., \& Oliva-Martínez, J. (2012). Dificultades de comprensión de nociones relativas a la clasificación periódica de los elementos químicos : la opinión de profesores e investigadores en educación química [Difficulties in learning about the periodic table: the opinion of teachers and resea. Revista Científica, 16(2), 5371. https://doi.org/10.14483/23448350.4023

Hernández-Sampiere, R., Fernández-Collado, C., \& Baptista-Lucio, P. (2014). Metodología de la investigación. [Investigation methodology] (Sexta). McGraw-Hill Education. https://n9.cl/65f

López-Guerrero, M. del M., López-Guerrero, G., \& Rojano-Ramos, S. (2018). Uso de un simulador para facilitar el aprendizaje de las Reacciones de Óxido-Reducción. Estudio de caso en la Universidad de Málaga [Use of a simulator to facilitate learning of the oxide-reductions. Study of case at the university of Málaga]. Educación Química, 29, 79-98. https://doi.org/10.22201/fq.18708404e.2018.3.63728

Marcano-Godoy, K. (2020). Estrategias didácticas para la enseñanza y aprendizaje de "los elementos químicos y su información en la tabla periódica" [Didactic strategies for the teaching and learning of "the chemical elements and their information in the periodic table"]. Revista Educación Las Américas, 10, 84-105. https://doi.org/10.35811/rea.v10i0.96 
Plutin-Pacheco, N., \& García-López, A. (2016). Estrategia didáctica basada en la lúdica para el aprendizaje de la química en la secundaria básica cubana [Didactic strategy based on fun for learning chemistry in Cuban elementary school]. Revista Cubana de Química, 28(2), 610-624. https://n9.cl/qsebz

Ramos-Mejía, A. (2020). ¿Cómo se puede usar el célular como pretexto para enseñar la tabla periódica? [How can the cell phone be used as an excuse to teach the Periodic Table?] Educacion Quimica, $31(1)$, 49-61. https://doi.org/10.22201/fq.18708404e.2020.1.70399

Rojas-Viteri, J., Álvarez-Zurita, A., \& Bracero-Huertas, D. (2021). Uso de Kahoot como elemento motivador en el proceso enseñanza-aprendizaje [Use of Kahoot as a motivating element in the teaching-learning process]. Cátedra, 4(1), 98-114. https://doi.org/10.29166/catedra.v4i1.2815

Tejada-Tovar, C., Chicangana-Collazos, C., \& Villabona-Ortiz, Á. (2013). Enseñanza de la química basada en la formación por etapas de acciones mentales (caso enseñanza del concepto de valencia) [Teaching of Chemistry Based on Training by Stages of Mental Actions (Teaching case of Valence Concept)]. Revista Virtual Universidad Católica Del Norte, 38, 143-157. https://revistavirtual.ucn.edu.co/index.php/RevistaUCN/article/view/410 